Decano Facultad de Ciencias Médicas. UNC

Prof. Dr. Marcelo A Yorio

Vice Decana Facultad de Cs. Médicas. UNC

Prof. Dra. Marta Fiol de Cuneo.

Secretaría de Salud Pública y Ambiente

Prof. Dr Gustavo Irico

Directora de la Revista

Prof. Dra. Ma. Cristina Cometto

\section{Comité editorial}

Prof. Mg. María Borsotti

Escuela de Salud Pública. FCM UNC

Prof. Mg. Oscar Mareca

Esc. de Salud Pública. FCM UNC

Prof. Dra. Patricia F. Gómez

Escuela de Enfermería FCM UNC.

Prof. Med. Gustavo Martínez

Municipalidad de Córdoba

Prof. Mg. Rubén Castro Toschi

Esc. de Salud Pública. FCM UNC

Prof. Mg. Abelardo Rahal

Escuela de Salud Pública. FCM UNC

\section{Comité de Redacción}

Prof. Dr. Leandro Dionisio

Esc. de Salud Pública. FCM UNC

Med. Mariela Roldan

Hospital Nacional de Clínicas

Mg. Susana Rivolta

Esc. de Salud Pública FCM UNC

Dr. Ariel Abeldaño

Esc. de Salud Pública FCM UNC

Dra. Monica Bella

Esc. de Salud Pública FCM UNC

Mg. Hugo L. Pizzi

Esc. de Salud Pública FCM UNC

\section{Consejo Científico Nacional}

Prof. Dra. Noemí Bordoni. Directora del Instituto de Investigaciones en Salud Pública. Coordinadora Ejecutiva de la Maestría en Salud Pública. UBA

Prof. Dra. Norma Cristina Meichtry. Doctor of Philosophy (Sociología- Demografía). Profesora Titular Ordinaria en Geografía Argentina UNNE. Directora Instituto de Revista de Salud Pública, (XIX) 1:1, abr

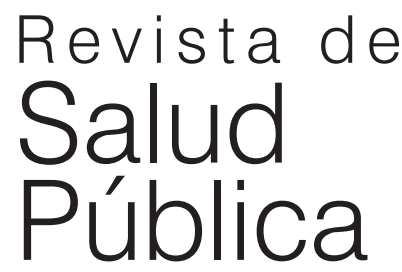

2015 Investigaciones Geohistóricas. Investigadora Independiente en el Inst. de Investigaciones Geohistóricas. CONICET

Dr. Jorge Kiguen Prof. Titular Ordinario de Epidemiologia. Universidad Nacional del Litoral. Rep. A.

Prof. Mg Ana M Heredia. Profesor Titular Regular Escuela de Enfermería. Departamento de Ciencias Sociales. Universidad Nacional de Quilmes

Dra. Silvina Malvarez. Ex consultora de la OPS OMS WDC

Dr. Luis Simes. Instituto Universitario de Ciencias de la salud. Universidad Barceló. Bs. As.

Dr. Bruno Sebastián Ribotta. Investigador Asistente del CONICET. Lugar de trabajo Centro de Investigaciones y Estudio sobre Cultura y Sociedad. CIECS, CONICET y UNC. Universidad Nacional de Córdoba.

\section{Consejo Científico Internacional}

Prof. Dr. Giorgio Solimano. Universidad de Chile

Dr. Charles Godue. Ex consultor OPS. WDC

Prof. Dr. Juan Jesús Gestal Otero. Universidad de Sgo. de Compostela. España

Prof. Dr. José Ramón Martínez Riera. Profesor Titular del Depto. de Enfermería Comunitaria. Medicina Preventiva y Salud Pública e Historia de la Ciencia de la Universidad de Alicante. España. 
La Revista de Salud Pública es una publicación temática, indizada, con arbitraje externo de pares nacionales e internacionales, trimestral, destinada a la publicación de la producción técnico científica relacionada al área de la salud y en especial de la Salud Pública. Editada por la Escuela de Salud Pública de la Facultad de Ciencias Médicas de la Universidad Nacional de Córdoba.

Consta de ISSN 1853-1180 para la versión Impresa y ISSN 1852-9429 para la Electrónica.

The Journal of Public Health is a thematic publication, indexed, external peer refereed national and international, biannual, for the publication of the technical-scientific production related to the area of health and in particular Public Health. Published by the School of Public Health, Faculty of Medical Sciences of the University National Córdoba, ISSN $1853-1180$ consists of the printedversion and for Electronic ISSN 1852-9429.

Bibliotecóloga: Mónica S. Perfetti

Traducción al inglés: Andrea Ternengo

Traducción al portugués: Osvaldo J. Casero

Tesorería y Finanzas: Cdora. Soledad Marquez

Diseño y Diagramación: Vladimir López Barrios

Escuela de Salud Pública. Calle Enrique Barros esq. Enf. Gordillo Gomez CP 5000. Córdoba. República Argentina. Te: 0351 - 4334042 / 4333023

www.saludpublica.fem.unc.edu.ar saludpublica@fcm.unc.edu.ar publicacionesp@fcm.unc.edu.ar ISSN 1853-1180

Las responsabilidades por los juicios, opiniones, puntos de vista, o traducciones; expresados en los artículos publicados corresponden exclusivamente a los autores.

No está permitida la reproducción parcial o total del contenido de la revista, sin la previa autorización de los editores de la Revista de Salud Pública.

Impreso en:

Taller General de Imprenta, UNC Abril, 2017

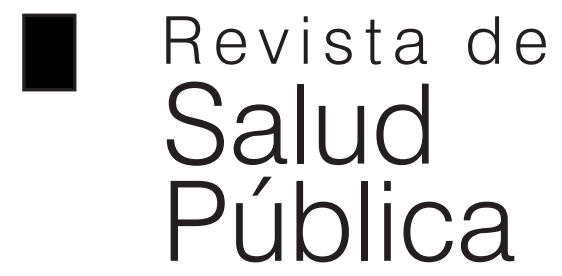




\section{Sumario}

\section{EDITORIAL}

\section{ARTICULOS CIENTIFICOS}

Perfil de consumo de alimentos durante la jornada escolar en una comunidad educativa vulnerabilizada de la ciudad de Córdoba, Argentina.

Giordano, María S., Barnetche, María M., Álvarez, Pedro S., Cornejo, Lila S.

16 Ácaros párásitos en pestañas y párpados humanos. Análisis de prevalencia de demodex folliculorum en consultas oftalmológicas en la ciudad de Córdoba, Argentina

Maffrand, Roque A., Pizzi, Hugo L., Pizzi, Rogelio D., Maffrand, Carola M., Tomas, Axel

25 Características del proceso de la maternidad en la provincia del Neuquén durante el periodo 1995/2014. La importancia del parto humanizado.

Álvarez, María E., Bercovich, Gabriela, Zanovello, Estefanía.

35 Modalidades de atención y actividades en salud mental orientadas a niñas y sus familias. Estudio descriptivo en los sistemas públicos de salud de Rio Negro y Neuquén. Periodo 2014-2016.

Dra. Parra Marcela A., Mg. De Gregorio María G., Esp. Gallosi Lorena, Esp. Morales Silvia, Lic. Novellino Ximena,Lic. Bercovich Gabriela, Psic. Ale Moira

46 Alimentos consumidos por preescolares como colación y su relación con el estado nutricional.

Oberto María G., Schiaroli Jessica A., Lencina Rocío del Cielo., Sosa Mariela I., Sanchéz Roberto J.

\section{UNA MIRADA HISTORICA}

De la "revista del círculo médico de córdoba" a la "revista médica de córdoba": un mercado publicitario de medicamentos e insumos médicos en el interior nacional. Córdoba, Argentina, 1912-1938.

\section{FORO ABIERTO DE OPINION}

El trabajo multidisciplinario en salud pública. La aportación de una mirada antropológica

\section{Summary}

\section{EDITORIAL}

\section{SCIENTIFIC ARTICLES}

Food consumption profile during school day in a vulnerable school community in the city of Cordoba, Argentina.

Giordano, María S., Barnetche, María M., Alvarez, Pedro S., Cornejo, Lila S.

Parasitic mites in human eyelashes and eyelids. Prevalence analysis of demodex folliculorum in ophthalmological consultations in the city of Cordoba, Argentina.

Maffrand, Roque A., Pizzi, Hugo L., Pizzi, Rogelio D., Maffrand, Carola M., Tomas, Axel

Characteristics of the maternity process in the province of Neuquen during the period 1995/2014.the importance of humanized childbirth.

Álvarez, María E., Bercovich, Gabriela, Zanovello, Estefanía

Type of care and mental health activities oriented to girls and their families. Descriptive study in public health systems in Rio Negro and Neuquen. Period 2014-2016

Dra. Parra Marcela A., Mg. De Gregorio María G., Esp. Gallosi Lorena, Esp. Morales Silvia, Lic. Novellino Ximena,Lic. Bercovich Gabriela, Psic. Ale Moira.

Snacks eaten by pre-school children and its relation to nutritional state.

Oberto María G., Schiaroli Jessica A., Lencina Rocío del Cielo., Sosa Mariela I., Sanchéz Roberto J.

\section{A HISTORIC LOOK}

From "revista del círculomédico de córdoba" to "revista médica de córdoba": advertising market of drugs and medical supplies in the interior of the country. Cordoba, Argentina, 1912-1938.

\section{OPEN FORUM OF OPINION}

Multi-disciplicary work in public health. The contribution of an anthropological look. 


\section{Revista indizada en base de datos}

\section{EDITORIAL}

\section{ARTIGOS CIENTIFICOS}

Perfil do consumo de alimentos durante uma jornada escolar em uma comunidade educacional vulnerável da cidade de Córdoba, Argentina.

Giordano, María S., Barnetche, María M., Álvarez, Pedro S., Cornejo, Lila S.

16 Ácaros parasitas em cílios e pálpebras humanas. Análise da prevalência do demodex folliculorum em consultas oftalmológicas, na cidade de Córdoba, Argentina.

Maffrand, Roque A., Pizzi, Hugo L., Pizzi, Rogelio D., Maffrand, Carola M., Tomas, Axel

25 Características do processo da maternidade na província de Neuquén durante o período de 1995/2014. A importância do parto humanizado.

Álvarez, María E., Bercovich, Gabriela, Zanovello, Estefanía.

35 Padrões de cuidados e ações de saúde mental voltados para meninas e suas famílias. Estudo descritivo em sistemas de saúde pública de Río Negro e Neuquén. Período de 2014-2016.

Dra. Parra Marcela A., Mg. De Gregorio María G., Esp. Gallosi Lorena, Esp. Morales Silvia, Lic. Novellino Ximena,Lic. Bercovich Gabriela, Psic. Ale Moira.

46 Alimentos consumidos por préescolarizados como lanche e sua relação com o estado nutricional.

Oberto María G., Schiaroli Jessica A., Lencina Rocío del Cielo., Sosa Mariela I., Sanchéz Roberto J.

56 UM OLHAR HISTÓRICO

Da «revista del círculo médico de córdoba» para «revista médica de córdoba»: um mercado de publicidade de medicamentos e suprimentos médicos no interior nacional. Cordoba, Argentina, 1912-1938.

\section{FORUM ABERTO OPINIÃO}

O trabalho multidisciplinar em saúde pública. A contribuição de um olhar antropológico

\section{REGRAS PARA TRABALHOS}

\section{LILACS}

Literatura Latinoamericana y del Caribe en Ciencias de la Salud. Bireme, Sistema Latinoamericano y del Caribe de Información en Ciencias de la Salud, Brasil. http://www. bireme.br/

\section{CUIDEN}

Base de Datos Bibliográfica sobre Cuidados de Salud en Iberoamérica. Base de datos de enfermería en español, Granada - España http://www.doc6.es/index/

\section{PERIODICA}

Base de datos de la Dirección General de Bibliotecas de la UNAM. Universidad Nacional Autónoma de México. http://132.248.9.1:8991/F/-/?func=find-b0\&local_base=PER01

\section{RENICS}

Red Nacional de Información en Ciencias de la Salud. http://www.renics.com.ar/

\section{DOAJ}

Directory of open access journals Universidad Lund, Suecia http://www. doaj.org/

NÚCLEO BÁSICO DE REVISTAS CIENTÍFICAS ARGENTINAS. CAYCITCONICET. R. Argentina HTTP://www. caicyt-conicet.gov.ar/nucleo-basico-derevistas-cientificas/

SISTEMA NACIONAL DE REPOSITORIOS DIGITALES - Biblioteca Electrónica de Ciencia y Tecnología del Ministerio de Ciencia, Tecnología e Innovación Productiva (Mincyt). Se accede a través de este link:

http://www.biblioteca.mincyt.gob.ar/revistas/index?letter $=\&$ title $=$ revista + de + salud + publica\&openaccess $=$ false \&gran_area $=3 \&$ area $=\&$ view $=$ list $\& y t 0=$ Busca

Revistas de la Universidad Nacional de Córdoba espacio destinado a revistas electrónicas, de acceso librema la produccion cientifica, un recurso publico, es por ello que somos parte de este sitio que la universidad ofrece de acceso libre a la produc

El sistema con que opera este sitio es Open Journal Systems (OJS).

Se accede: http://revistas.unc.edu.ar/ 